\title{
Stereoselective Reactions with Chiral Schiff Base Metal Complexes
}

\author{
Andrea Gualandia , Claire Margaret Wilson ${ }^{\mathrm{b}}$, and Pier Giorgio Cozzi ${ }^{\star a}$
}

This paper is dedicated to the memory of Professor T. Katsuki, a pioneer in Salen chemistry

\begin{abstract}
Chiral Schiff bases are privileged chiral ligands, capable of transmitting chiral information in many stereoselective processes. Their modular nature, the possibility to modify their electronic character, and their ability to coordinate many metals, gives these chiral ligands broad utility and these have been investigated by many research groups around the world. For more than 20 years our research group has devoted attention to the application of chiral Schiff bases in the development of new stereoselective processes and in this brief survey we present our principal results and achievements in this field.
\end{abstract}

Keywords: Catalysis · Lewis acids · Metal Complexes $\cdot$ Schiff Bases · Stereocontrol

\section{Introduction}

The development of stereoselective reactions presents a formidable task for which multifaceted expertise is necessary. The design of effective chiral ligands to control the regio-, diastereo-, and enantioselectivity is key to this task. In the past, serendipity and intuition aided pioneers in this field. More recently, the design of new chiral ligands is helped by a deeper understanding of the transmission of stereochemical information at the molecular level. ${ }^{[1]}$ The challenges posed by the desire to control chirality at the molecular level have resulted in major breakthroughs in chemical catalysis ${ }^{[2]}$ and have conversely influenced the comprehension of the chiral information transfer in molecular systems. ${ }^{[3]}$ The major efforts in asymmetric catalytic synthesis have had an enormous economic and scientific return, which are difficult to fully quantify. ${ }^{[4]}$ In the past few decades, a wide variety of asymmetric catalytic transformations have been invented, most of them relying on particularly well defined classes of chiral ligands. Chiral ligands capable of performing well in several catalytic asymmetric transformations were defined 'privileged ligands' by Jacobsen

${ }^{\star}$ Correspondence: Prof. P. G. Cozzi E-mail: piergiorgio.cozzi@unibo.it

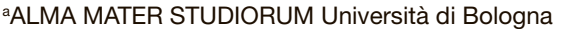
Dipartimento di Chimica "G. Ciamician" Via Selmi 2, 40126 Bologna, Italy bUniversity of Bath

Department of Chemistry

Claverton Down, Bath, BA2 7AY, UK
(Fig. 1). ${ }^{[5]}$ This class of privileged ligands includes phosphoroamidites, ${ }^{[6]}$ BINOL, ${ }^{[7]}$ TADDOL, ${ }^{[8]}$ PHOX, ${ }^{[9]} \mathrm{BOX},{ }^{[10]}$ and many others. From the versatile and readily accessible class of chiral Schiff bases, Salen ligands have emerged as one of the most successful in transmitting chiral information. [11] The modular structure allows for specific variation of the ligand framework.

The acid-base properties of the metal-ligand arrangement can be specifically tuned to catalyse important asymmetric transformations. In addition, in many catalytic reactions promoted by Schiff base metal complexes, supramolecular formation of 'special pair' catalysts are involved in electrophile-nucleophile bi-functional activation (Fig. 2). ${ }^{[12]}$ Due to these properties, Salen base metal complexes frequently show extraordinary levels of stereocontrol.
Salen ligands were established as versatile and effective ligands in $1869,{ }^{[13]}$ with the first systematic studies conducted in the 1930s by Pfeiffer and co-workers. ${ }^{[14]}$ These workers introduced the first non-racemic Salen ligand. Salen metal complexes are indeed considered privileged catalysts as demonstrated by their successful application in many challenging asymmetric reactions. Their straightforward preparation and their modular structure allows a wide structural modification (Scheme 1) enabling their adaption for many asymmetric transformations. The peculiar arrangement of Salen metal complexes and the combination of multiple catalytic sites (which act to enforce an intramolecular cooperative reaction pathway) results in enhanced reaction rates and higher selectivity. Salen ligands are readily obtained by condensation reaction between an aldehyde and an

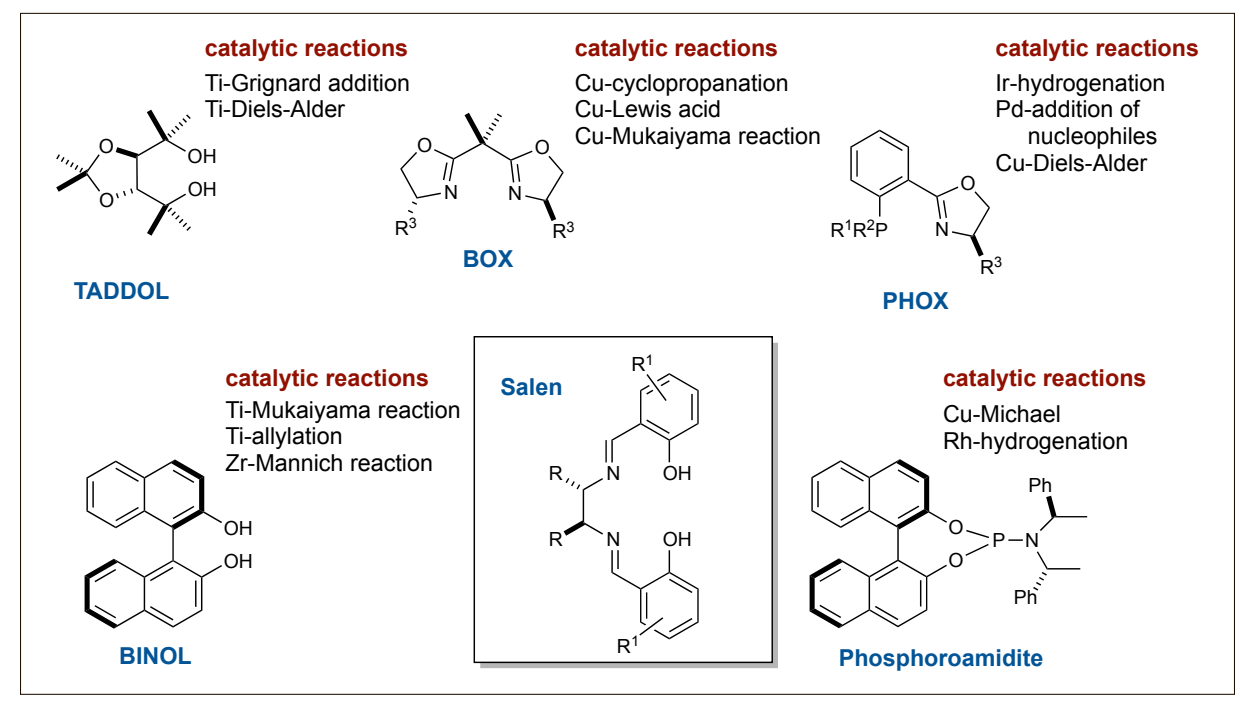

Fig. 1. Privileged ligands and their catalytic reactions. 


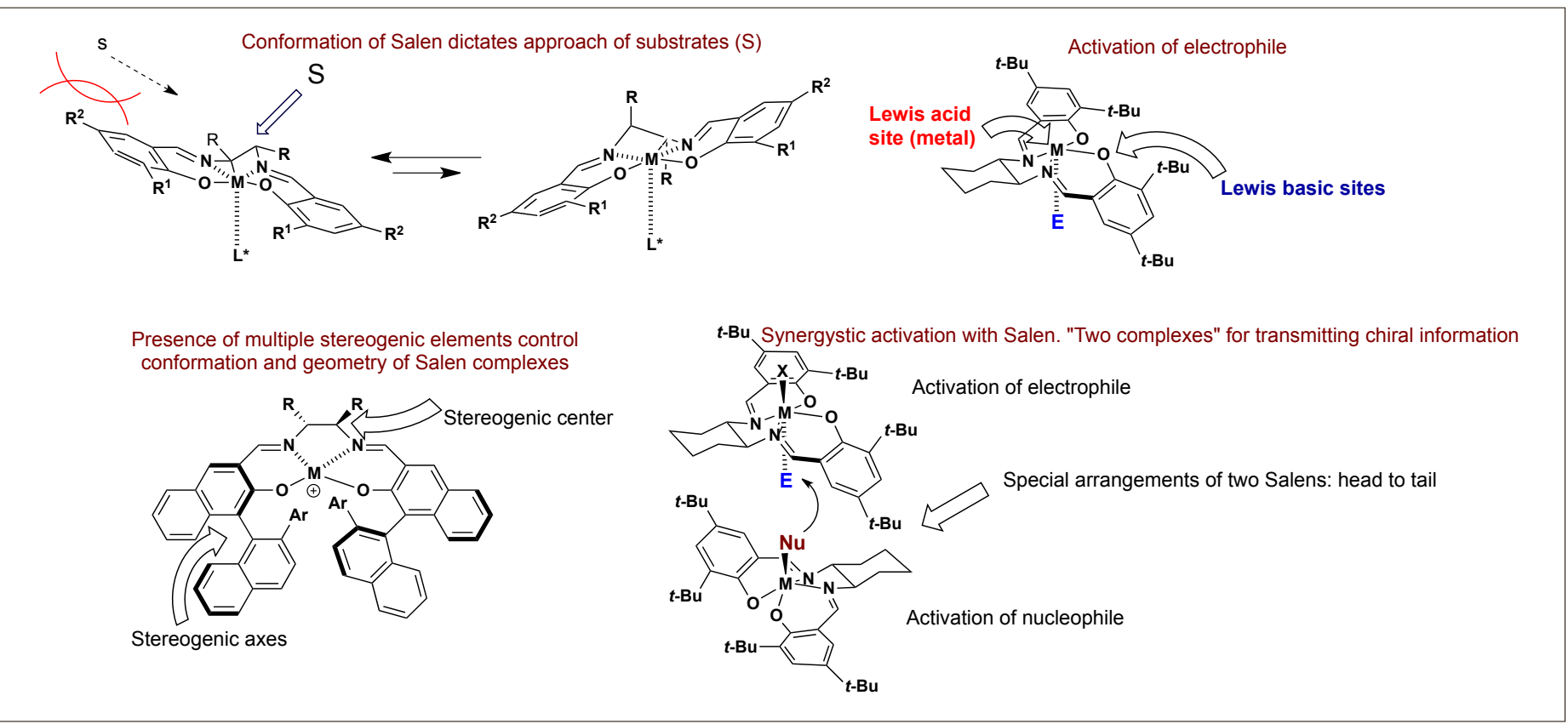

Fig. 2. Transmission of chiral information via properties of Salen ligands. Clockwise: Conformation (Stepped); Stereogenic elements; Head to tail; Acid-base properties.

amine, performed in the presence of dehydrating agents (Scheme 1A). ${ }^{[15]}$

\subsection{Transmission of Chiral Information by Salen Complexes}

In the straightforward synthesis of Salen ligands the diamine plays a delicate role in transmitting the chiral information to the metal complexes. 1,2-Diaminocyclohexane, 1,2-diamino-1,2-diphenylethane, and 1,1'-binaphthyl-2,2'-diamine (Fig. 3A) are commercially available and the corresponding Schiff base can be prepared without difficulty. It is possible to introduce more stereogenic elements in a Salen moiety. Matsumotu demonstrated this in work on the stereoselective epoxidation of non-functionalized olefins, ${ }^{[16]}$ in which the chiral Salen ligand had stereocenters in the ethylendiamine and in the aromatic moieties. In doing so, a second generation of Salens were designed (Fig. 3B). The corresponding manganese complexes gave excellent results in the epoxidation of cis-olefins. In most studies the Salen ligand is made by reaction of 1,2-diamines with a variety of substituted salicylaldehydes. The axial substituents in the diamine backbone are hydrogens. However, by coordinating one of the substituents in the diamine backbone to the metal, the other substituent can be forced to occupy the axial position. As the axial substituent is in proximity to the metal coordinating site, it is ideally positioned to affect the stereoselective binding

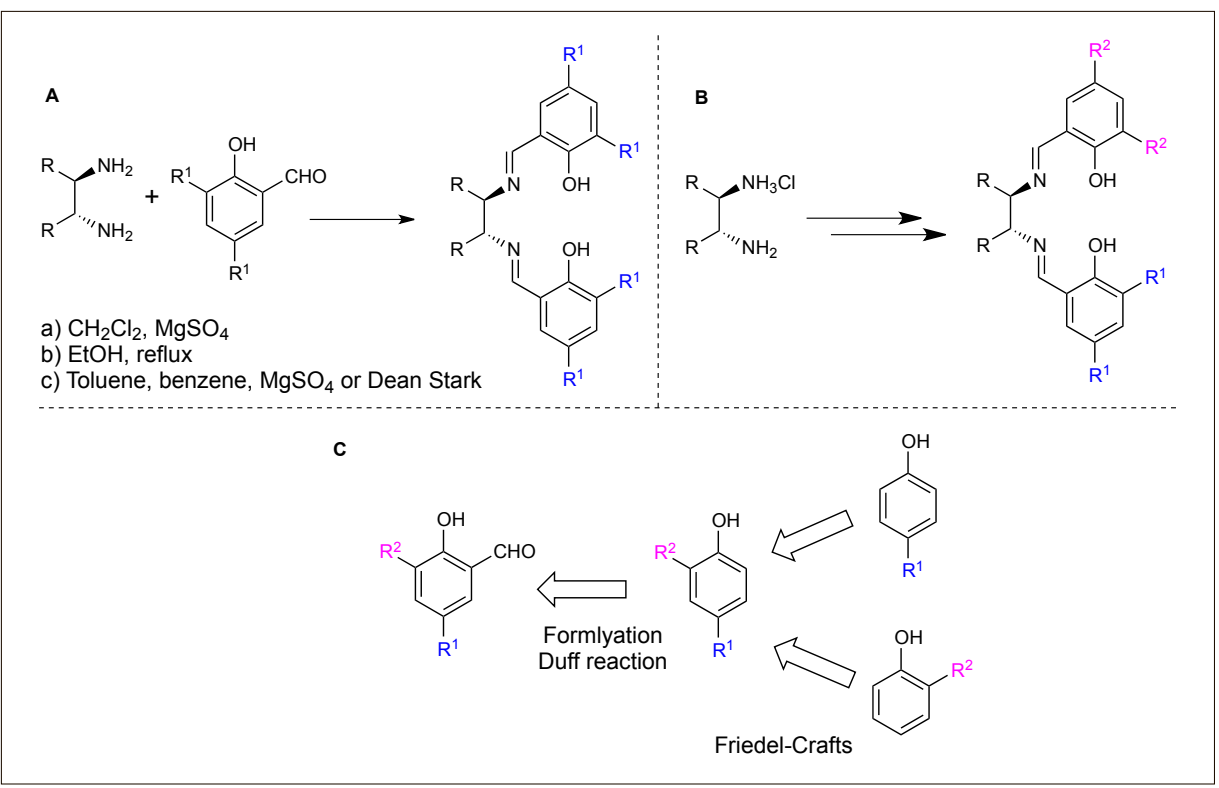

Scheme 1. Preparation of salicyl aldehydes and their Salen ligands. of the substrate. For Salen complexes an octahedral M(Salen) can exist in three potential configurations, trans (planar), cis-a and cis-b. Due to the rigidity of the Salen ligand, the trans-geometry is more accessible for $\mathrm{M}(\mathrm{Salen})$. Coordination of a bidentate ligand forces the $\mathrm{M}$ (Salen) complex to adopt a cis-configuration. If the cis-coordination is adopted, the metal become stereogenic and the corresponding $\mathrm{M}($ Salen$)$ complexes can exist in diastereoisomeric forms. Particularly with imine and amine ligands (by reduction of one of two imine moieties present in Salen), cis-coordination of many metal complexes was obtained, as seen in Salalen and Salan ligands. ${ }^{[17]}$ Salen metal complexes bearing chiral diamines can exist in two distinct forms. The substituent on the diamine can be axial or equatorial. Steric hindrance among the diamine substituents can influence the equilibria between the two conformations. However, the two conformations appear quite accessible to the Salen metal complexes in solution. If the diamine has coordinating groups, this ligation of the group can force the equilibrium towards one conformer.

\subsubsection{Preparation of Salen Metal Complexes}

Guidelines for the complexation steps in the preparation of the different Salen ligands were suggested in 2004 and are still valid today (Scheme 2).[11d] Salen metal complexes are often used as catalysts in the presence of other metal salts or organometallic reagents and in such conditions the stability of the metal complex needs to be considered. However, there are examples in the literature of trans-metalation reactions using Salen complexes in the presence 
of other metals. ${ }^{[18]}$ Kleji and co-workers have studied the subject in detail, showing that under certain conditions Salen metal complexes can exchange metal ions. This problem limits the stability of the metal complexes in catalytic mixtures of metals. [19] In many reactions coordination of the nucleophile/electrophile or dimerization are involved. Clearly, the double activation mechanism or establishment of the supramolecular role of the Salen ligands are still processes requiring investigation. In many reactions involving $\mathrm{M}($ Salen) metal complexes, three distinct mechanistic pictures can be considered: i) Coordination and activation of substrates by Lewis acid properties of the metallo Salen complexes can induce several transformations. ii) The bifunctional coordinative properties of the M(Salen) metal complexes can assemble a nucleophile and an electrophile together when they are in close proximity. On such activation, supramolecular aspects of the Salen coordination chemistry are important. In addition, multimetallic Salen complexes are often used to enhance the double activation. iii) An important mechanistic consideration that needs to be considered in many Salen mediated transformations is

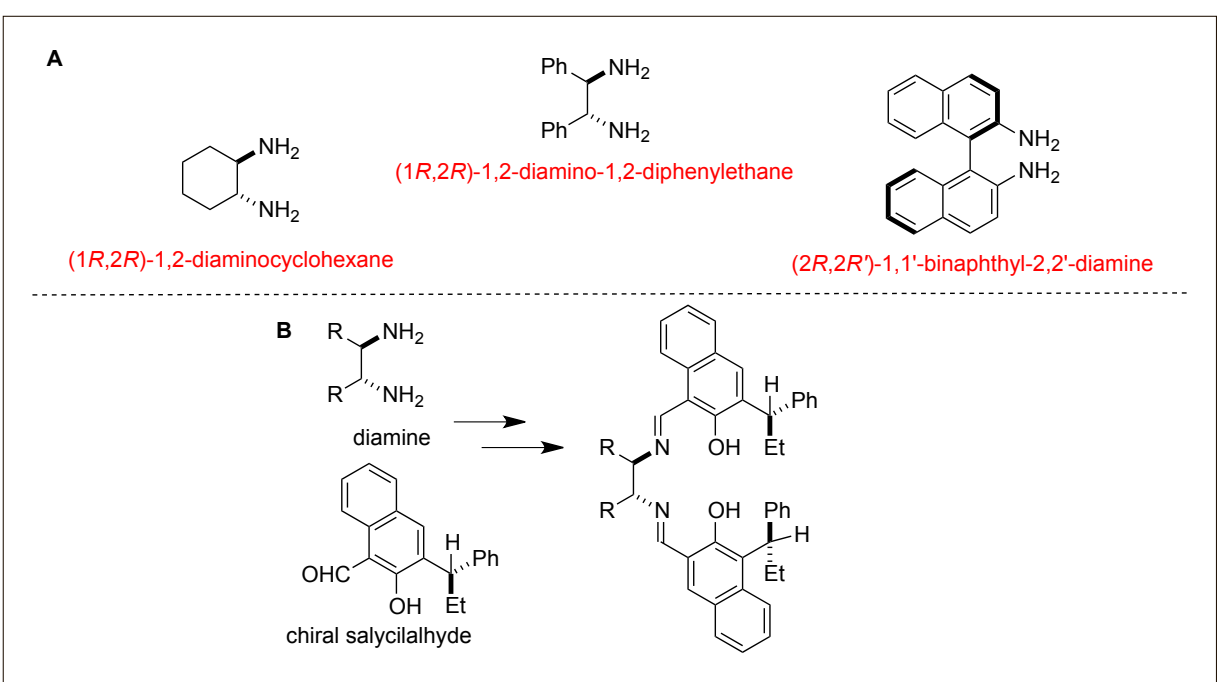

Fig. 3. Commonly employed chiral diamines and an example of chiral salicylic aldehyde for the synthesis of chiral Salen ligands.

that different Salen units can act together, forming a sort of 'special pair' in which nucleophiles and electrophile are again activated by the $\mathrm{M}($ Salen $)$ complexes.

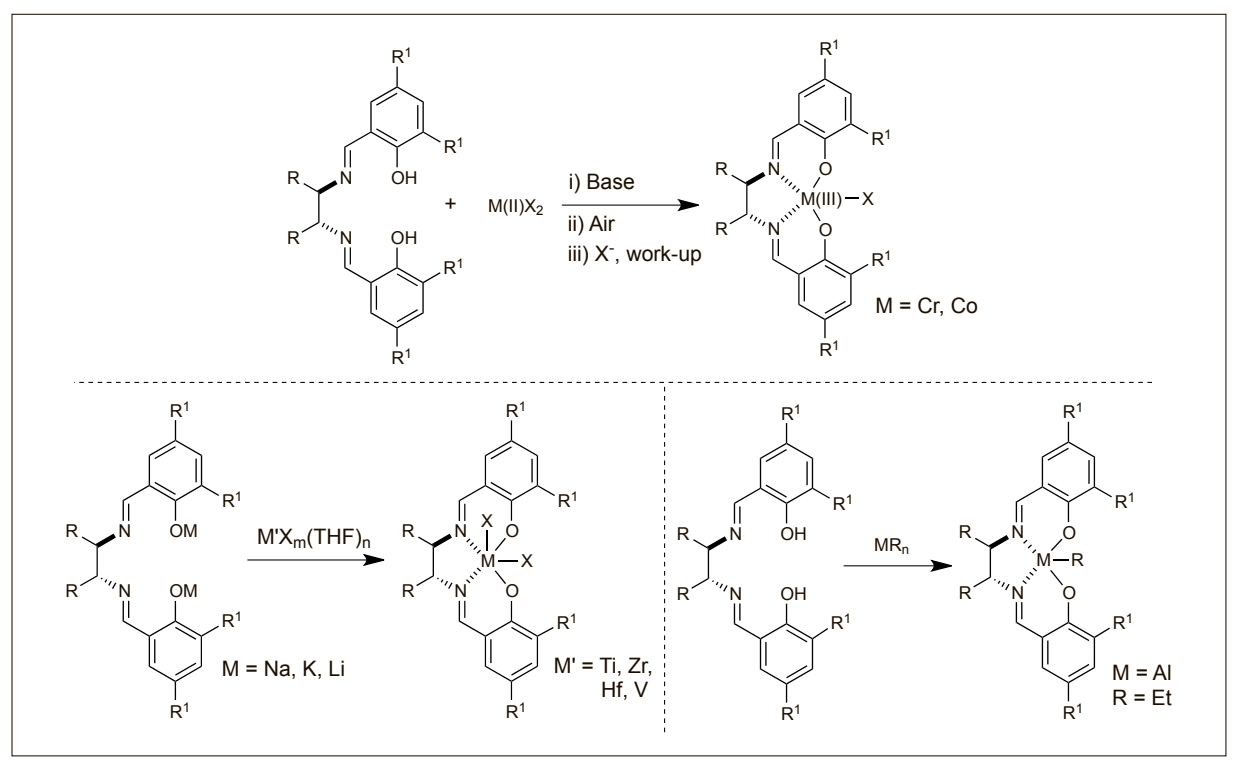

Scheme 2. Simple strategies for the preparation of Salen metal complexes.

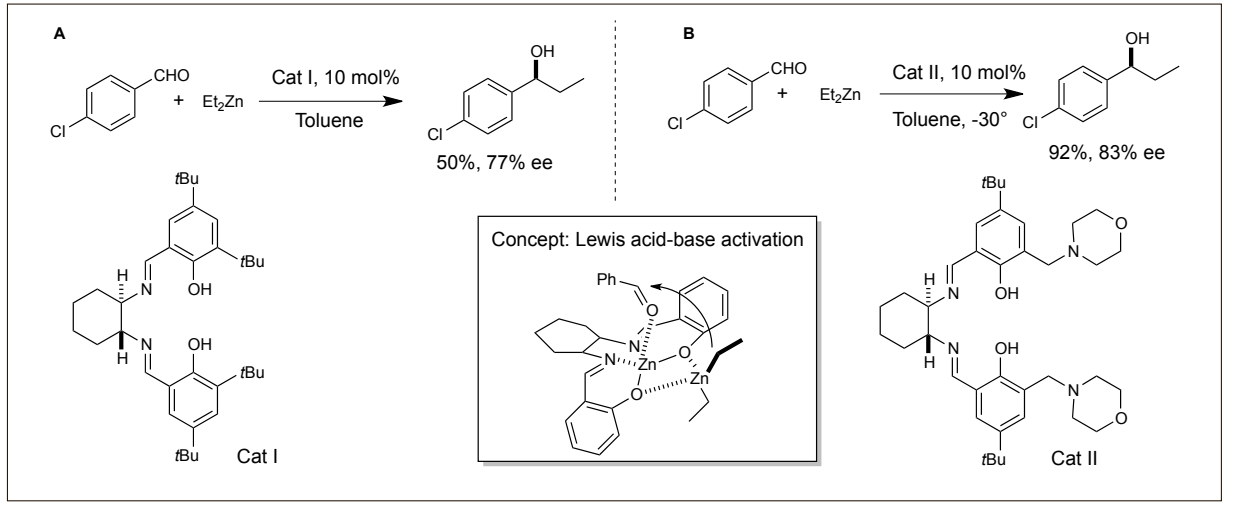

Scheme 3. Addition of $\mathrm{Et}_{2} \mathrm{Zn}$ to aldehydes promoted by $\mathrm{Zn}$ Salen metal complex.

\section{Different Concepts for the Application of Salen Complexes in Stereoselective Reactions}

Over the last 20 years our group has applied several concepts to Salen metal catalysis in the discovery of new stereoselective transformations, following the principles discussed above. Although a detailed and complete mechanistic picture of the effective role played by Salen complexes in these and other reactions is far from completely established, nevertheless these principles can help in the development, understanding and establishing of new reactions.

\subsection{Addition of Organometallic Reagents, the 'Organizational' Role of Salen Complexes.}

Salen metal complexes were reported to be able to coordinate metal ions and metal complexes. ${ }^{[20]}$ This ability can also be extended to organometallic reagents. The oxygen atoms present in the Salen moiety can coordinate Lewis acidic reagents, changing their reactivity. This is crucial in the case of organozinc reagents, as these reagent are of moderate reactivity. Metal complexes that can coordinate the zinc reagents and enhance the electrophilicity of carbonyls present synergistic cooperation necessary to control the reactivity of organozinc.[21] Goedken reported crystal structures of bimetallic alkyl systems where $\mathrm{Et}_{2} \mathrm{Zn}$ is able to coordinate the oxygen group in Salen metal complexes. ${ }^{[22]}$ We used this concept to develop methods for the addition of $\mathrm{Et}, \mathrm{Zn}$ to aldehydes, with moderate results (Scheme 3A). ${ }^{[23]}$ Better yields and stereoselectivity were realized by Kozlowski using a modified Salen ligands structure in which modular bifunctional Salen catalysts containing Lewis acid and Lewis base activating groups were 
present. ${ }^{[24]}$ The functional groups are able to coordinate $\mathrm{Et}_{2} \mathrm{Zn}$, with tertiary amines inserted at appropriate distances from the Lewis acidic zinc center. (Scheme 3B).

The cooperative coordination properties of Salen metal were found to be suitable to promote the addition of alkynes to ketones. ${ }^{[25]}$ In general, the formation of tertiary alcohols by addition of organometallic reagents to ketones poses formidable challenges, particularly due to the differences in reactivity of ketones in comparison with aldehydes and imines. ${ }^{[26]}$ The lack of hydrogen substituents on ketones poses problems for the control of coordination geometry, avoiding ordered transition states. Nevertheless, assuming a model in which alkynylzinc (formed in the reaction mixture) is activated and coordinated by the oxygen of the $\mathrm{Zn}$ (Salen), moderate to good stereoselection is observed in the reaction (Scheme 4A). As a linear correlation was found between the enantiomeric excess of the Salen ligand and that of the product, this suggested that just one molecule of the Salen catalyst is involved in the enantio-differentiating step. Using theoretical investigations and careful mechanistic analysis, Jacobsen found that in many reactions promoted by Salen metal complexes, ${ }^{[27]}$ two molecules of Salen are involved. One molecule of Salen activates the nucleophile and the other molecule of Salen activates the electrophile, acting in cooperative manner with a particular arrangement of the two Salen molecules, referred to as head-to-tail (Fig. 2). ${ }^{[27]}$ In the alkynylation of ketones, the cooperative role played by Salen is absent, with the Salen acting as a bifunctional catalyst. While the addition of phenylacetylene and $\mathrm{Et}_{2} \mathrm{Zn}$ does not pose problems with $\mathrm{Zn}$ (Salen), other organometallic reagents were found to be less activated. In particular, $\mathrm{Me}_{2} \mathrm{Zn}$ is rather unreactive, and few cases of the addition of $\mathrm{Me}_{2} \mathrm{Zn}$ have been reported. ${ }^{[28]}$ We found that $\mathrm{ClCr}$ (Salen) promoted the addition of $\mathrm{Me}_{2} \mathrm{Zn}$ at room temperature, affording excellent results (Scheme 4B). ${ }^{[29]}$ The process was particularly useful in the case of aromatic aldehydes, as the enantiomeric excesses obtained were in the range of 92-99\%, with good yields. Interestingly it was possible to use challenging hindered aldehydes, with good yields and enantiomeric excesses obtained.

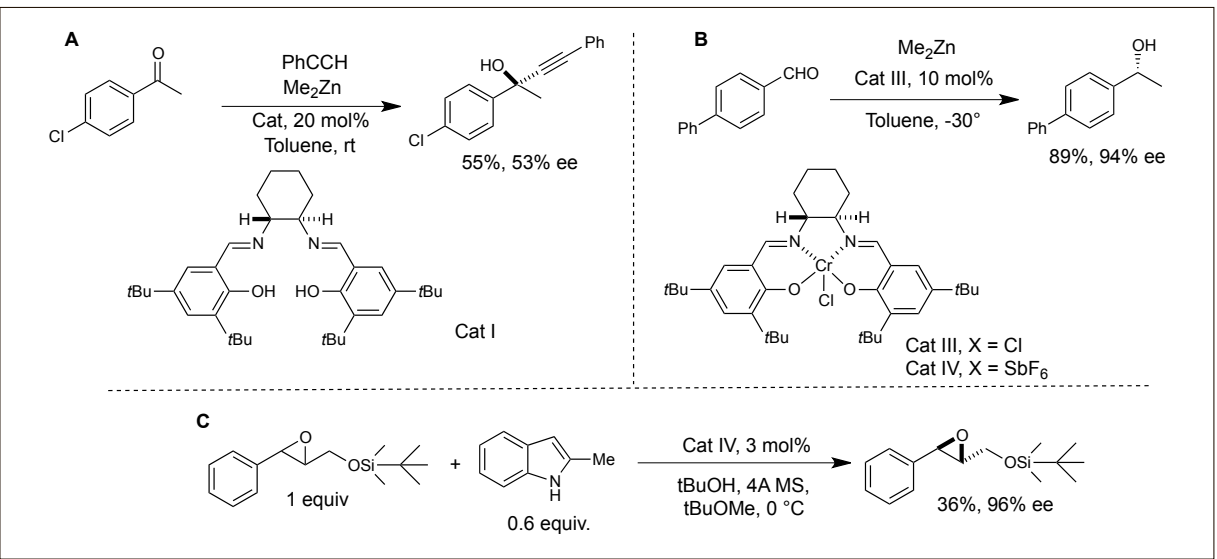

Scheme 4. A) Addition of PhCCZnMe promoted by chiral Zn(Salen). B) CICr(Salen) as chiral catalysts for useful transformations. C) Friedel-Crafts-type addition of indoles to epoxides.

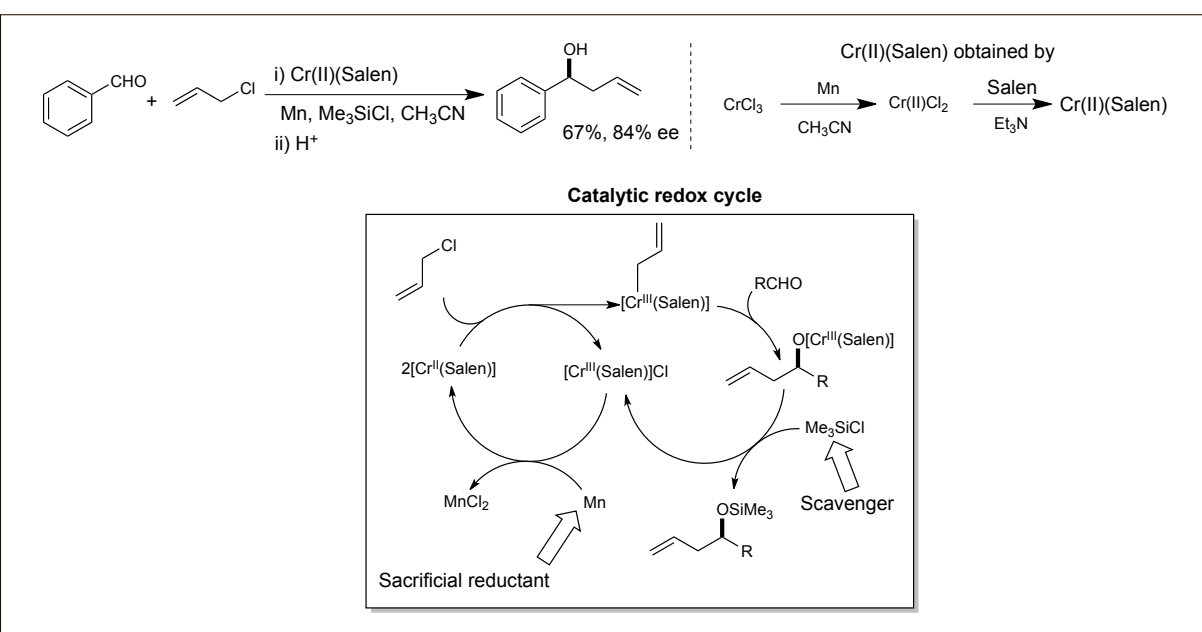

Scheme 5. Application to the Nozaki-Hiyama-Kishi reaction. The enantioselective redox-reaction promoted by Salen complexes.

\subsection{Activation of Nucleophiles and Kinetic Resolution}

Enantioenriched epoxides can be obtained by kinetic resolution. ${ }^{[30]}$ The fundamental work of Jacobsen in developing $\mathrm{ClCr}(\text { Salen })^{[31]}$ and $\mathrm{Co}(\text { Salen })^{[32]}$ kinetic resolution of epoxides enhanced the understanding of the particular properties and the development of reactions mediated by Salen. ${ }^{[33]}$ Jacobsen investigated the Lewis acidic properties of Salen complexes for the creation of a chiral environment to dictate nucleophilic attacks on epoxides. However, 1,2-disubstituted epoxides were rather difficult substrates for such transformations. The kinetic resolution of cis and trans substituted aromatic epoxides was reported by our research group using a Friedel-Crafts-type addition of indoles to epoxides, ${ }^{[34]}$ giving a general method for the preparation of both cis and trans aromatic epoxides with high ee values starting from racemic substrates (Scheme 4C).

\subsection{Salen Ligands in Catalytic Redox Reactions}

Salen metal complexes are redox active complexes, capable of undergoing redox chemistry at the metal. ${ }^{[35]}$ In 2006 Fürstner reported the concept of catalytic redox systems, applying a sacrificial reductant $(\mathrm{Mn})$ and a scavenger $\left(\mathrm{Me}_{3} \mathrm{SiCl}\right)$ to a Nozaki-Hiyama-Kishi reaction. Previously conducted with an over-stoichiometric amount of highly sensitive $\mathrm{Cr}(\mathrm{II}) \mathrm{Cl}_{2}$, this variant allowed for the utilization of the chromium source in catalytic qualities. In principle, chromium complexes with appropriate chiral ligands could be used to introduce enantiocontrol in the Nozaki-HiyamaKishi reaction. With the ability to stabilize metals in different oxidation states and the ability to store electrons, Salen ligands were investigated in the enantioselective variant of Nozaki-HiyamaKishi reactions (Scheme 5). In 1999 we successfully reported the first catalytic enantioselective Nozaki-Hiyama-Kishi reaction. ${ }^{[36]}$ The reaction of crotylchromium reagent showed a peculiar simple syn stereoselection, ${ }^{[37]}$ and in nonlinear effect studies an uncommon negative effect was recorded. The reaction is thought to proceed via a molecular aggregate in which more than one $\mathrm{Cr}$ (Salen) unit is involved. Due to the syn stereoselection obtained with crotyl reagents, an acyclic transition state is hypothesized, with two $\mathrm{Cr}$ (Salen) molecules; one behaving as a Lewis acid, coordinating the aldehyde and another bearing the organometallic chromium reagent. From these pioneering studies, many efficient catalysts and processes were developed for the Nozaki-HyiamaKishi reaction. ${ }^{[38]}$ 


\subsection{Radical Reactions and Salen Metal Complexes}

In 2005 we introduced a stereoselective one-pot three-component $\mathrm{Ni}$ (II) catalyzed imino Reformatsky reaction. ${ }^{[39]}$ We have extended the pioneering work of Adrian, ${ }^{40]}$ who discovered an efficient, nickel-catalyzed Reformatsky-type three-component condensation that combines aldehyde, 2-methoxyaniline, and bromocarbonyl reagent (esters, ketones, and amides) in the presence of excess $\mathrm{Me}_{2} \mathrm{Zn}$ (Scheme 6A). The mechanism involved the reduction of the $\mathrm{Ni}(\mathrm{II})$ complex to a $\mathrm{Ni}(0)$ complex, oxidative addition of the bromoester to the $\mathrm{Ni}(0)$ complex, and a $\mathrm{Ni}(\mathrm{II}) / \mathrm{Zn}$ (II) exchange which leads to an organozinc Reformatsky reagent. In many reactions tested in the presence of $N$-methylephedrine (an inexpensive chiral ligand) we obtained moderate to good enantiomeric excesses. However, the ligand was used in greater than stoichiometric quantities. In 2006 we reported the first practical catalytic enantioselective catalytic Reformatsky reaction with ketones (Scheme 6B). ${ }^{[41]}$ To avoid the use of nickel salts more reactive iododerivates were used, as it is reported that zinc enolates can be prepared by direct exchange of iodo esters with Et $\mathrm{Zn}_{2}{ }^{[42]}$ To avoid the background non-stereoselective reaction the less reactive $\mathrm{Me}_{2} \mathrm{Zn}$ was employed, in attempts to catalyze a metal complex exchange. The use of $\mathrm{ClMn}($ Salen) afforded moderate stereoselectivity with acetophenone in the presence of an excess of $\mathrm{Me}_{2} \mathrm{Zn}$. With further studies we discovered that zinc enolates are generated by $\mathrm{Me}_{2} \mathrm{Zn}$ via a radical pathway[43] in which an oxidant acts as the initiator. ${ }^{[4]}$ Although a detailed mechanistic investigation is necessary in order to fully conclude that radical species are involved in these catalytic reactions, only $\mathrm{ClMn}$ (Salen) activates the reaction, under strictly anaerobic

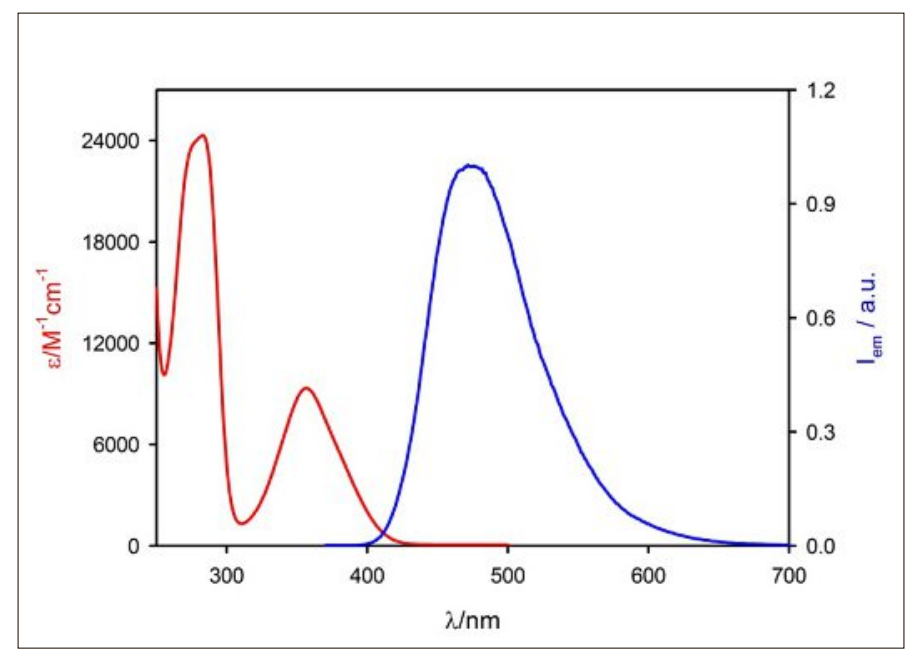

Fig. 4. Absorption spectrum (red solid line) and emission spectrum (blue solid line, $\lambda_{\text {exc }}=340 \mathrm{~nm}$ ) of $\mathrm{CIAI}\left(\right.$ Salen) in $\mathrm{CH}_{3} \mathrm{CN}$ at room temperature. Optical pathlength $1 \mathrm{~cm}$ conditions. It is probable that Salen metal complexes are able to initiate the reaction via a single electron transfer (SET) mechanism. ${ }^{[45]}$

\subsection{Salen Metal Complexes and Photoredox Catalysis}

The area of photoredox catalysis has attracted considerable interest ${ }^{[46]}$ since the pioneering work published by MacMillan in 2008. ${ }^{[47]}$ Ruthenium and iridium bipyridyl complexes are used as photocatalysts, due to their specific properties. ${ }^{[48]}$ Such catalysts absorb visible light and become strong reductants or oxidants. Their ability to transfer electrons can be exploited in radical reactions. ${ }^{[49]}$ The major problems associated with these two metals are high cost and low abundance. Attempts to use more abundant metals in photocatalysis, such copper, ${ }^{[50]}$ chromium, ${ }^{[51]}$ or iron ${ }^{[52]}$ have been reported. Some years ago, in collaboration with the photo-physicists of our department, ${ }^{[53]}$ we studied the photophysical properties of Salen metal complexes. From this seminal contribution, many other photophysical studies were

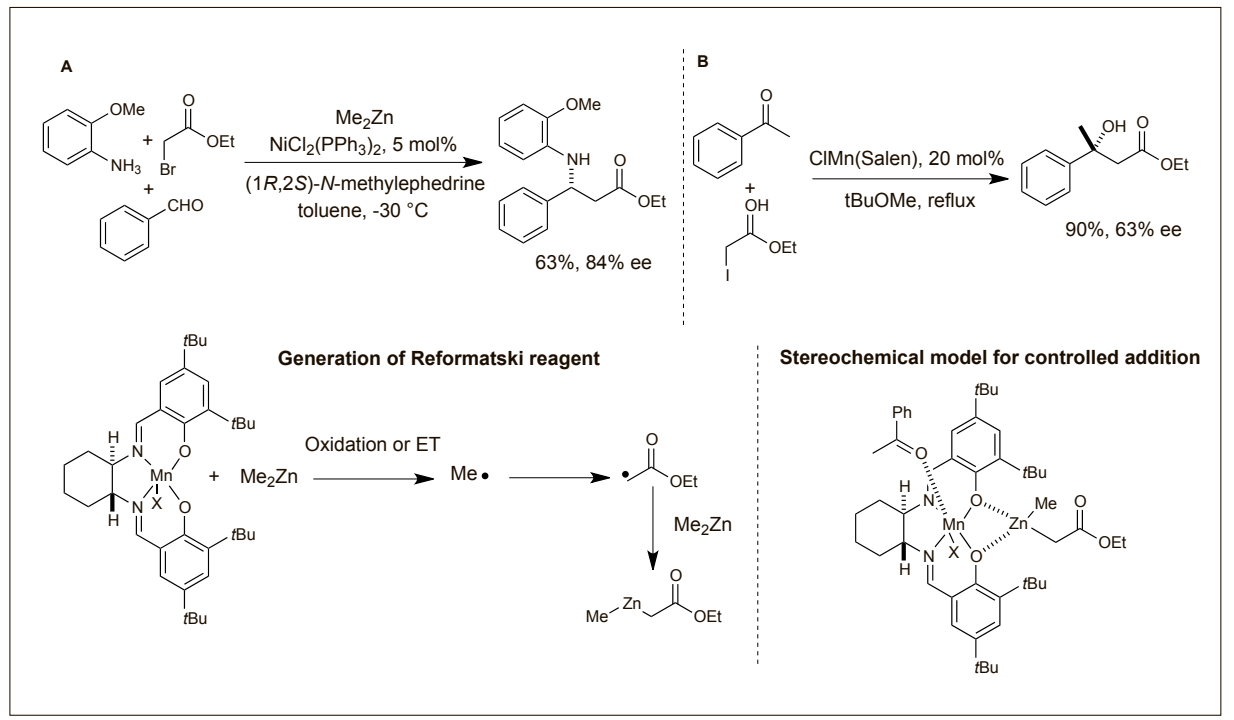

Scheme 6. Radical reaction promoted by Mn(Salen). devoted to Salen and their behavior as sensors or in other interesting applications. ${ }^{[54]}$ Recently, we have taken advantage of our studies to develop new photoredox reactions using cheap and abundant Salen metal complexes (Fig. 4). ${ }^{[55]}$

\section{Conclusions}

Over the past 20 years, we have investigated the use of Salen metal complexes to explore new reactivity and concepts. The application of Salen complexes in new transformations is still possible, as we have recently discovered and discussed briefly in this survey. As the focus turns to the use of more abundant and cheaper metals, these types of complexes are expected to gain more attention. As it is possible to have synergistic and cooperative catalysis mediated by Salen complexes, increased attention to the synthesis of new chiral Salen ligands is predicted. Finally, the possibility to explore achiral Salen complexes in combination with ACDC (asymmetric counter ion catalysis) or bioconjugation increases the possibility to discover and develop even more useful asymmetric transformations.

\section{Acknowledgements}

All co-workers of the studies mentioned in this review are gratefully acknowledged for their substantial practical and intellectual contributions. Bologna University, Fondazione Del Monte, SLAMM project are acknowledged for financial support to Andrea Gualandi.

Received: July 26, 2017

[1] P. Walsh, M. C. Kozlowski, 'Fundamentals of Asymmetric Catalysis', University Science Book, 2009.

[2] B. Cornils, W. A. Herrmann, R. Schlögl, C.-H Wong, 'Catalysis from A to Z', Wiley-VCH, Weinheim/New York, 2000.

[3] W. Herrmann, B. Cornils, Angew. Chem. Int Ed. Engl. 1997, 36, 1049. 
[4] I. Ojima, 'Catalytic Asymmetric Synthesis', III ed, 2010.

[5] T. P. Yoon, E. N. Jacobsen, Science 2003, 299, 1691.

[6] J. F. Teichert, B. L. Feringa, Angew. Chem., Int Ed. 2010, 49, 2846.

[7] J. M. Brunel, Chem. Rev. 2005, 105, 857.

[8] a) H. Pellissier, Tetrahedron 2008, 64, 10279; b) D. Seebach, A. K. Beck, A. Heckel, Angew. Chem., Int. Ed. 2001, 40, 92.

[9] G. Helmchen, A. Pfaltz, Acc. Chem. Res. 2000 , 33, 336 .

[10] A. Pfaltz, 'Bisoxazolines - a privileged ligand class for asymmetric catalysis', in 'Asymmetric Synthesis', 2nd ed, Ed. I. Ojima, 2008.

[11] a) L. Canali, D. C. Sherrington, Chem. Soc. Rev. 1999, 28, 85; b) J. F. Larrow, E. N. Jacobsen, Top. Organomet. Chem. 2004, 6, 123; c) T. Katsuki, Adv. Synth. Catal. 2002, 344, 131; d) P. G. Cozzi, Chem. Soc. Rev. 2004, 33, 410; e) S. J. Wezenberg, A. W. Kleij, Angew. Chem., Int. Ed. 2008, 47, 2354; f) E. M. McGarrigle, D G. Gilheany, Chem. Rev. 2005, 105, 1563; g) T. Katsuki, Coord. Chem. Rev. 1995, 140, 189; h) Carlos Baleizão, H. Garcia, Chem. Rev. 2006 106, 3987.

[12] D. J. Dixon, Beilst. J. Org. Chem. 2016, 12 , 1089 , and articles in this issue.

[13] H. Schiff, Ann. Suppl. 150, 1869, p. 193

[14] P. Pfeiffer, W. Christeleit, T. Hesse, H. Pfitzinger, H. Theilert, J. Prakt. Chem. 1938, 150, 261.

[15] S. Srimurugan, P. Suresh, B. Babu, H. N. Pati, Mini Rev. Org. Synth. 2008, 5, 228.

[16] a) R. Irie, K. Noda, Y. Ito, M. Matsumotu, Tetrahedron Lett. 1990, 31, 7335; b) R. Irie, K. Noda, Y. Ito, M. Matsumotu, Tetrahedron Lett. 1991, 32, 1555.

[17] K. Matsumoto, B. Saito, T, Katsuki, Chem. Commun. 2007, 3619.

[18] D. Aufustin, G. Rima, H. Gornitzka, J. Barrau, Organometallics 2000, 19, 4276

[19] E. C. Escudero-Adan, J. Benet-Buchholz, A. W. Kleij, J. Chem. Soc. Dalton Trans. 2008, 734.

[20] S. Matsunaga, M. Shibasaki, Chem. Commun. 2014, 50, 1044, and ref. therein.

[21] 'Lewis Bases Catalysis in Organic Synthesis', Eds. E. Vedejs, S. Denmark, Wiley-VCH, 2016.

[22] S. J. Dzugan, V L. Goedken, J. Organomet. Chem. 1988, 356, 249.
[23] P. G. Cozzi, A. Papa, A. Umani-Ronchi, Tetrahedron Lett. 1996, 37, 4613.

[24] E. F. Di Mauro, M. C. Kozlowski, Org. Lett. 2001, 3, 3053.

[25] P. G. Cozzi. Angew. Chem., Int. Ed. 2003, 42, 2859.

[26] M. Shibasaki, M. Kanai, Chem. Rev. 2008, 108, 2853.

[27] D. D. Ford, L. P. C. Nielsen, S. J. Zuend, C. B. Musgrave, E. N. Jacobsen, J. Am. Chem. Soc. 2013, 135, 15595.

[28] J. Escorihuela, M. Isabel Burguete, G. Ujaque, A. Lledós, S. V. Luis, Org. Biom. Chem. 2016, 14, 11125, and refs therein.

[29] P G. Cozzi, P. Kotrusz, J. Am. Chem. Soc. 2006, $128,4940$.

[30] J. M. Keith, J. F. Larrow, E. N. Jacobsen. $A d v$. Synth. Catal. 2001, 343, 5.

[31] L. E. Martinez, J. L. Leighton, D. H. Carsten, E. N. Jacobsen, J. Am. Chem. Soc. 1995, 117, 5897.

[32] M. Tokunaga, J. F. Larrow, F. Kakiuchi, E. N. Jacobsen, Science 1997, 277, 936.

[33] E. N. Jacobsen, Acc. Chem. Res. 2000, 33, 421.

[34] M. Bandini, P. G. Cozzi, P. Melchiorre, A. Umani Ronchi, Angew. Chem., Int. Ed. 2004, 43, 84

[35] Y. Shimazaki, 'Oxidation Chemistry of Metal(II) Salen-Type Complexes', in Electrochemistry, Ed. M. Khalid, 2013, InTech, DOI: 10.5772/48372. Available from: https:// www.intechopen.com/books/electrochemistry/ oxidation-chemistry-of-metal-ii-Salen-typecomplexes

[36] a) M. Bandini, P. G. Cozzi, P. Melchiorre, A. Umani-Ronchi, Angew. Chem., Int. Ed. 1999, 38, 3357 b) M. Bandini, P. G. Cozzi, A. UmaniRonchi, Angew. Chem., Int. Ed. 2000, 39, 2327. c) M. Bandini, P. G. Cozzi, S. Morganti, A. Umani-Ronchi, Org. Lett. 2001, 3, 1153; d) M. Bandini, P. G. Cozzi, A. Umani-Ronchi, Tetrahedron 2001, 57, 835; e) M. Bandini, P. G. Cozzi, A. Umani-Ronchi, Polyhedron 2000, 19, 537; f) M. Bandini, P. G. Cozzi, P. Melchiorre, R. Tino, A. Umani-Ronchi, Tetrahedron: Asymm. 2001, 12, 1063 .

[37] Normally, because a cyclic Zimmerman-Traxler type of transition state (in which aldehyde and allyl reagent are coordinated by chromium atom) is favored, anti-configurated products are observed in Nozaki-Hiyama-Kishi reactions.
[38] G. C. Hargaden, P. J. Guiry, Adv. Synth. Catal. 2007, 349, 2407.

[39] P. G. Cozzi, E. Rivalta, Angew. Chem., Int. Ed. 2005, 44, 3600 .

[40] a) J. C. Adrian, Jr., M. L. Snapper, J. Org. Chem. 2003, 68, 2143; b) J. C. Adrian, Jr., J. L. Barkin, R. J. Fox, J. E. Chick, A. D. Hunter, R. A. Nicklow, J. Org. Chem. 2000, 65, 6264.

[41] a) P. G. Cozzi, Angew. Chem., Int. Ed. 2006 45, 2951; b) P. G. Cozzi, A. Mignogna, L. Zoli, Synthesis 2007, 2746.

[42] a) K. Kanai, H. Wakabayashi, T. Honda, Org. Lett. 2000, 2, 2549; b) K. Kanai, H. Wakabayashi, T. Honda, Heterocycles 2002, 58, 47.

[43] E. Mileo, F. Benfatti, P. G. Cozzi, M. Lucarini, Chem. Commun. 2009, 469.

[44] P. G. Cozzi, A. Mignogna, L. Zoli, Pure Appl. Chem. 2008, 80, 891 .

[45] T. Storr, E. C. Wasinger, R. C. Pratt, T. D. P. Stack, Angew. Chem., Int. Ed. 2007, 46, 5198.

[46] C. K. Prier, D. A. Rankic, D. W. C. MacMillan, Chem. Rev. 2013, 113, 5322.

[47] D. A. Nicevicz, D. W. C. MacMillan, Science 2008, 322, 77.

[48] V. Balzani, G. Bergamini, P. Ceroni, Angew. Chem., Int. Ed. 2015, 54, 11320.

[49] A. Studer, D. P. Curran, Angew. Chem., Int. Ed. 2016, 55,58

[50] S. Paria, O. Reiser, ChemCatChem 2014, 6, 2477.

[51] L. A. Büldt, O. S. Wenger, Angew. Chem., Int Ed. 2017, 56, 5676, and ref. therein.

[52] A. Gualandi, M. Marchini, L. Mengozzi, M. Natali, M. Lucarini, P. Ceroni, P. G. Cozzi, ACS Catal. 2015, 5, 5027.

[53] The department is dedicated to Giacomo Ciamician, a prophet of photochemistry, the study of which he introduced to our department. For seminal and important contributions, see: G. Ciamician, 'The Photochemistry of the Future', Science 1912, 36, 385.

[54] K.-T. Yeung, W.-P. To, C. Sun, G. Cheng, C. Ma, G. S. M. Tong, C. Yang, C.-M. Che, Angew. Chem., Int. Ed. 2017, 56, 133, and ref. therein.

[55] A. Gualandi, L. Mengozzi, H. Testfay, A. Franc, M. Marchini, P. Ceroni, P. G. Cozzi, manuscript in preparation. 